\title{
Patency of endoscopic ultrasound-guided gastroenterostomy in the treatment of malignant gastric outlet obstruction
}

\section{다)(1) $\odot$}

Authors

Janine B. Kastelijn¹, Leon M.G. Moons ${ }^{1}$, Francisco J. Garcia-Alonso², Manuel Pérez-Miranda², Viliam Masaryk², Uwe Will $^{3}$, Ilaria Tarantino ${ }^{4}$, Hendrik M. van Dullemen ${ }^{5}$, Rina Bijlsma ${ }^{6}$, Jan-Werner Poley ${ }^{7}$, Matthijs P. Schwartz ${ }^{8}$, Frank P. Vleggaar $^{1}$

Institutions

1 Department of Gastroenterology and Hepatology, University Medical Center Utrecht, Utrecht, The Netherlands

2 Department of Gastroenterology and Hepatology, Hospital Universitario Rio Hortega, Valladolid, Spain

3 Department of Gastroenterology and General Internal Medicine, SRH Wald-Klinikum, Gera, Germany

4 Gastroenterology and Endoscopy Unit, Mediterranean Institute for Transplantation and Advanced Specialized Therapies (IsMeTT), Palermo, Italy

5 Department of Gastroenterology and Hepatology, University Medical Center Groningen, Groningen, The Netherlands

6 Department of Gastroenterology and Hepatology, Martini Hospital Groningen, Groningen, The Netherlands

7 Department of Gastroenterology and Hepatology, Erasmus University Medical Center, Rotterdam, The Netherlands

8 Department of Gastroenterology and Hepatology, Meander Medical Center, Amersfoort, The Netherlands

submitted 25.2.2020

accepted after revision $\quad 28.5 .2020$

Bibliography

DOI https://doi.org/10.1055/a-1214-5659 |

Endoscopy International Open 2020; 08: E1194-E1201

(c) Georg Thieme Verlag KG Stuttgart · New York eISSN 2196-9736

Corresponding author

Prof. Dr. F.P. Vleggaar, Department of Gastroenterology and Hepatology, University Medical Center Utrecht, P.O. Box 85500, 3508 GA, Utrecht, The Netherlands.
Fax: +0031887550724

f.vleggaar@umcutrecht.nl

\section{ABSTRACT}

Background and study aims Endoscopic ultrasoundguided gastroenterostomy (EUS-GE) with a lumen-apposing metal stent (LAMS) is a novel, minimally invasive technique in the palliative treatment of malignant gastric outlet obstruction (GOO). Several studies have demonstrated feasibility and safety of EUS-GE, but evidence on long-term durability is limited. The aim of this study was to evaluate patency of EUS-GE in treatment of malignant GOO.

Patients and Methods An international multicenter study was performed in seven centers in four European countries. Patients who underwent EUS-GE with a LAMS between March 2015 and March 2019 for palliative treatment of symptomatic malignant GOO were included retrospectively. Our main outcome was recurrent obstruction due to LAMS dysfunction; other outcomes of interest were technical success, clinical success, adverse events (AEs), and survival.

Results A total of 45 patients (mean age $69.9 \pm 12.3$ years and $48.9 \%$ male) were included. Median duration of followup was 59 days (interquartile range [IQR] 41-128). Recurrent obstruction occurred in two patients $(6.1 \%)$, after 33 and 283 days of follow-up. Technical success was achieved in 39 patients (86.7\%). Clinical success was achieved in 33 patients $(73.3 \%)$. AEs occurred in 12 patients (26.7\%), of which five were fatal. Median overall survival was 57 days (IQR 32-114).

Conclusions EUS-GE showed a low rate of recurrent obstruction. The relatively high number of fatal AEs underscores the importance of careful implementation of EUSGE in clinical practice. 


\section{Introduction}

Malignant gastric outlet obstruction (GOO) can occur in up to $20 \%$ of patients with advanced or metastatic malignancies located in the distal stomach and peri-pancreatic region [1]. Accompanying obstructive symptoms such as vomiting and nausea are a burden to patients and quickly lead to malnutrition and poor performance status [2]. Given its advanced and often metastatic or unresectable stage, the main purpose of treating malignant GOO is palliation of these obstructive symptoms.

Traditionally, the two techniques to treat malignant GOO are endoscopic duodenal stent placement and surgical gastrojejunostomy (SG). Both interventions are effective palliative treatments with high success rates. Duodenal stent placement is minimally invasive and associated with favorable short-term results, such as fast relief of symptoms and improvement in food intake. However, a significant risk of late adverse events (AEs) was found, such as stent migration and recurrent obstruction, often necessitating reintervention [3-7]. SG] provides longerlasting patency with less need for reinterventions once the anastomosis is functioning and patients have recovered [3,8$10]$, but it is a more invasive treatment and associated with surgery-related morbidity such as gastroparesis or intestinal paralysis [11].

Endoscopic ultrasound-guided gastroenterostomy (EUS-GE) with a lumen-apposing metal stent (LAMS) is a novel technique for palliative treatment of malignant GOO. It seems to combine the advantages of duodenal stenting and SG], while potentially overcoming their shortcomings [12-16]. EUS-GE is minimally invasive and shows fast relief of symptoms, possibly with lower stent failure and reintervention rates than duodenal stenting $[12,13]$. It also avoids invasive surgery and related morbidity of SG], but might provide comparable long-term patency [14, 15].

Long-term patency of EUS-GE is crucial for maintaining oral intake and quality of life. However, current literature on durability of EUS-GE is limited. We aimed to assess recurrent obstruction and patency of EUS-GE in a multinational cohort of patients with malignant GOO.

\section{Patients and methods}

Patients with symptomatic malignant GOO who underwent EUS-GE with a LAMS were retrospectively included. Twenty-six European centers with known expertise in interventional endoscopy and LAMS were invited to participate in this study ( $\vee$ Fig. 1). In total, seven centers (six tertiary centers, one large regional center) from four countries (four centers from the Netherlands and one from Germany, Spain, and Italy) provided consecutive cases, from the first EUS-GE procedures performed in each hospital. Procedures were performed between March 2015 and March 2019. A subset of 10 patients has been reported previously [17]. Baseline characteristics (age, sex, obstructive symptoms, and diet tolerated before and after EUS-GE, etiology of malignant GOO, location of the obstruction, performance status, presence of ascites or peritoneal carcinomatosis, previous oncologic treatment); procedural characteristics
Invited European centres with LAMS expertise $(n=26)$

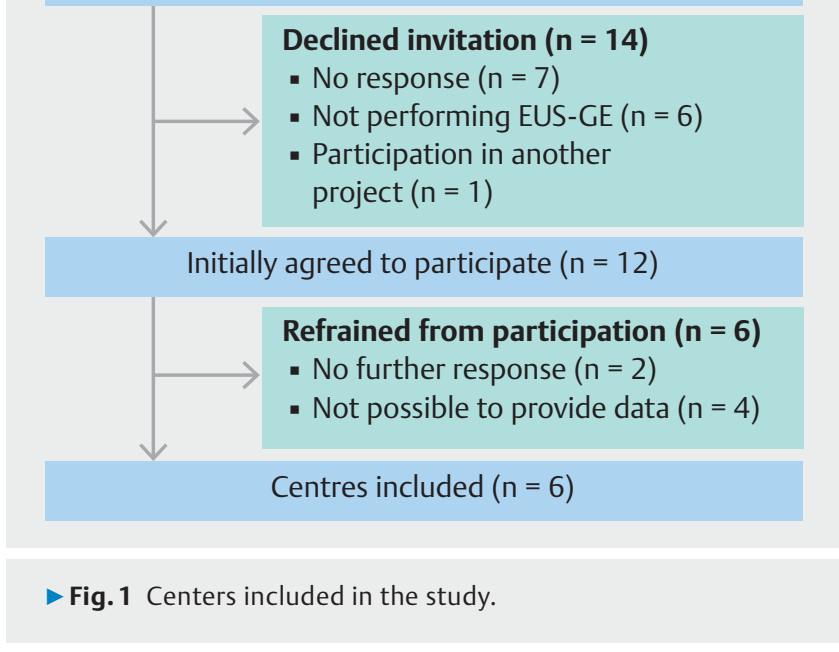

(year of intervention, method of stent placement, size of stent, balloon dilation of the stent, antibiotic administration), and follow-up data after EUS-GE (recurrent obstruction, technical and clinical success, time to oral intake, diet tolerated after technically successful EUS-GE, AEs, death, cause of death, duration of follow-up and survival) were retrospectively collected from electronic medical records. Data were stored in Castor EDC, a General Data Protection Regulation (GDPR) compliant Electronic Data Capture Tool.

Patients were eligible if they presented with symptomatic malignant GOO (nausea, vomiting, and intolerability of oral intake) due to obstruction confirmed endoscopically and/or by abdominal imaging, and had undergone EUS-GE with LAMS (Hot AXIOS Stent).

Additional information was requested from participating centers, general practitioners or treating physicians if data were missing or interpretation was hampered. Uncertainties about eligibility and outcome adjudication were discussed in a panel of two expert endoscopists from UMC Utrecht for consensus. For quality assurance, we excluded a series of 17 patients from one center, because in more than $50 \%$ of these patients eligibility could not be verified or follow-up was lacking. This study was approved by the Institutional Medical Research Ethics Committee of the University Medical Center Utrecht (MREC number 18/584) and complied with the GDPR at each participating institution. This study adhered to the Strengthening the Reporting of Observational Studies in Epidemiology (STROBE) Guidelines [18].

\section{EUS-GE procedural techniques}

EUS-GE procedures and per procedural care were performed according to local practice and endoscopists' preference. Either the direct puncture technique or the balloon-assisted technique was performed [19]. A gastroscope was introduced proximal to the stenosis and a feeding tube was placed distal to the stenosis through the working channel. The endoscope was then re- 
moved, followed by introduction of a linear array EUS endoscope. With the direct puncture technique, the jejunal or duodenal loop was identified by flushing saline with indigo carmine through the feeding tube into the post-stenotic duodenal-jejunal loop. With the balloon-assisted method, an extraction balloon was used for identification of the duodenal-jejunal loop. Identification of the loop could be confirmed with puncture and aspiration of luminal content with a 25G "finder needle." Subsequently, the loop was punctured using an electrocauteryenhanced delivery system and the $15 \times 10 \mathrm{~mm}$ or $20 \times 10 \mathrm{~mm}$ LAMS (Hot AXIOS Stent and Electrocautery-Enhanced Delivery System, Boston Scientific Corporation, United States) was deployed. Correct positioning and passage of the stent was confirmed fluoroscopically and endoscopically by backflow of the indigo-colored saline from the small intestine into the stomach. Subsequent balloon dilation of the LAMS was performed at the discretion of the endoscopist.

\section{Outcomes and definitions}

The main outcome was recurrent obstruction. It was defined as recurrence of GOO symptoms (nausea, vomiting, inability to tolerate oral intake) after a clinically successful EUS-GE, confirmed by endoscopy or abdominal imaging showing a dysfunctional LAMS.

Other outcomes of interest were as follows. Technical success was defined as adequate positioning and deployment of the LAMS, confirmed by endoscopy or fluoroscopy. Successful stent-in-stent placement of a second LAMS or a FCSEMS after the initial attempt failed was also considered technically successful. Clinical success was defined as the ability to tolerate at least a full liquid diet without vomiting, after a technically successful EUS-GE. The ability of oral food intake was measured by the Gastric Outlet Obstruction Scoring System (GOOSS) score as adapted by Adler et al: $0=$ no oral intake, $1=$ liquid intake only, 2 = soft solids, 3 =low residue/full diet [2]. AEs were defined as any AE occurring during EUS-GE or within the first month of follow-up suspected to be related to EUS-GE. Severity was graded according to the ASGE lexicon [20]. Survival was defined as the time between EUS-GE and death.

\section{Follow-up}

Patients were followed up until death or last contact as reported in the electronic patient files. Additional information about events during follow-up was requested from general practitioners or treating physicians if follow-up was missing or unclear. We considered patients free of recurrent obstruction if no recurrent obstructive symptoms were recorded at last contact (physical or by telephone) or before death.

\section{Statistical analysis}

Baseline characteristics are presented as means and standard deviations (SD) for normally distributed continuous variables and medians with interquartile range (IQR) for continuous variables with a skewed distribution. Absolute numbers and percentages are presented for categorical variables. Patients were censored at their last moment of contact if they were lost to follow-up. Due to the limited sample size, no subgroup analyses were performed. Statistical analyses were performed with STATA version 15.1 and $R$ version 3.5.1 was used for plotting the bar chart.

\section{Results}

\section{Baseline characteristics}

In total, 45 patients ( $48.9 \%$ male; mean age $69.9 \pm 12.3$ years) who underwent EUS-GE with a LAMS for symptomatic malignant GOO were included in this study. Twenty-one patients $(46.7 \%)$ presented with inability to tolerate oral intake (GOOSS 0 ) and $21(46.7 \%)$ tolerated liquids only (GOOSS 1). Nausea was reported in 27 patients $(62.8 \%)$ and vomiting in $38(88.4 \%)$. Etiologies of malignancies causing GOO are shown in Table 1. Fifteen patients (35.7\%) had ascites and 13 (33.3\%) had peritoneal carcinomatosis. Nine (23.7\%) had both ascites and peritoneal carcinomatosis. Seventeen patients (38.6\%) had not undergone oncological treatment, 16 (36.4\%) were previously treated with chemotherapy, 15 (34.1\%) received a duodenal stent, and four ( $9.1 \%$ ) had surgery within this anatomical region. These operations included a Whipple in two patients, pylorus-preserving pancreaticoduodenectomy in one and Roux-en-Y reconstruction in one ( $\triangleright$ Table 1$)$.

\section{Procedural characteristics}

The direct technique was used in 36 patients (80.0\%) and the balloon-assisted technique was applied in nine (20.0\%). The diameter of the deployed LAMS was $15 \mathrm{~mm}$ in 32 patients (72.7\%) and $20 \mathrm{~mm}$ in 12 patients (27.3\%). Balloon dilation immediately after stent placement was performed in 12 procedures (30.8\%). Pre-procedural antibiotics were administered to 18 patients $(40.0 \%)$ and post-procedural antibiotics were administered to 28 patients (62.2\%) ( $\triangleright$ Table 2 ).

\section{Outcomes}

\section{Recurrent obstruction}

Two of 33 patients $(6.1 \%)$ had recurrent obstruction after a clinically successful EUS-GE, occurring after 33 and 283 days ( $>$ Table 3). Median duration of follow-up in patients with clinical success was 73 days (interquartile range [IQR] 44-166). In both patients, repeat endoscopy was performed and showed a LAMS with a small luminal diameter and food impaction. The LAMS could not be passed with the endoscope. This was successfully solved endoscopically by removal of the food and balloon dilation. There were no signs of tissue in- or overgrowth. One additional patient underwent repeat endoscopy for recurrent obstructive symptoms after initial clinical success, but the LAMS was functional in this patient.

\section{Technical and clinical success}

Technical success was accomplished in 39 patients (86.7\%) ( $\triangleright$ Table 3). The six technical failures were related to stent misplacement in five patients, and to accidental puncture of a colonic loop with the finder needle and abortion of the procedure in one patient. Stent misplacement occurred initially in eight patients, but could be salvaged with a second LAMS (stent-in- 
- Table 1 Baseline characteristics.

Age, mean $\pm S D$

$69.9 \pm 12.3$

Male sex, n (\%)

$22(48.9)$

GOOSS score before EUS-GE

- Gooss 0 (no oral intake), n (\%)

$21(46.7)$

- GOOSS 1 (liquid only), n (\%)

$21(46.7)$

- Gooss 2 (soft solids), n (\%)

$3(6.7)$

- Gooss 3 (low residue/full diet)

Median GOOSS score before EUS-GE (IQR)

Obstructive symptoms, n (\%) ${ }^{1}$

- Nausea

$27(62.8)$

- Vomiting

$38(88.4)$

- Early satiety

15 (34.9)

- Anorexia

18 (41.9)

- Epigastric pain

$17(39.5)$

-Weight loss

$23(53.5)$

Aetiology, n (\%)

- Pancreatic cancer

$19(42.2)$

- Extrinsic/metastatic cancer ${ }^{2}$

$8(17.8)$

- Gastric cancer

$6(13.3)$

- Duodenal cancer

$3(6.7)$

- Periampullary cancer

$3(6.7)$

- Biliary tract cancer

$3(6.7)$

- Gall bladder cancer

2 (4.4)

- Lymphoma

$1(2.2)$

stent) in two patients and a FCSEMS (stent-in-stent) in one patient, resulting in a technically successful EUS-GE. Of the five patients in whom stent misplacement could not be salvaged, one patient underwent immediate surgical removal of the stent with closure of the perforation and four patients were managed endoscopically in the same session (duodenal stent placement in one patient; conservative treatment after removal of stent and closure with clip in three patients). Technical success was achieved in 10 of 13 patients (76.9\%) with peritoneal carcinomatosis and in 24 of 26 (92.3\%) without peritoneal carcinomatosis. Eleven of 15 patients (73.3\%) with ascites and 25 of 27 $(92.6 \%)$ without ascites were technically successful. Technical success was achieved in six of nine patients (66.7\%) with both peritoneal carcinomatosis and ascites.

Clinical success was achieved in 33 patients (73.3\%) who underwent EUS-GE (84.6\% of the patients who underwent a technically successful EUS-GE). Fifteen patients (38.5\%) were able to tolerate soft solids (GOOSS 2) and 18 (46.2\%) low residue/ full diet (GOOSS 3 ). Median time to resumption of oral intake was 1 day, ranging from 0 to 4 days. Of the five patients in whom EUS-GE was clinically unsuccessful, two died before hav-

\begin{tabular}{|c|c|}
\hline \multicolumn{2}{|l|}{ Location of obstruction, $\mathrm{n}(\%)^{3}$} \\
\hline - Antrum/pylorus & $10(22.7)$ \\
\hline - $1^{\text {st }} /$ superior part duodenum (bulb) & $14(31.8)$ \\
\hline - $2^{\text {nd }} /$ descending part duodenum & $17(38.6)$ \\
\hline - $3^{\text {rd }} /$ horizontal part duodenum & $7(15.9)$ \\
\hline Ascites, n (\%) & $15(35.7)$ \\
\hline Peritoneal carcinomatosis, n (\%) & $13(33.3)$ \\
\hline \multicolumn{2}{|l|}{ WHO performance status, n (\%) } \\
\hline - 0 & - \\
\hline - 1 & $11(25.6)$ \\
\hline-2 & $22(51.2)$ \\
\hline - 3 & $10(23.3)$ \\
\hline - 4 & - \\
\hline \multicolumn{2}{|l|}{ Previous oncologic treatment, $\mathrm{n}(\%)^{4}$} \\
\hline - None & $17(38.6)$ \\
\hline " Chemotherapy & $16(36.4)$ \\
\hline - Duodenal stent & $15(34.1)$ \\
\hline - Surgery ${ }^{5}$ & $4(9.1)$ \\
\hline \multicolumn{2}{|c|}{$\begin{array}{l}{ }^{1} \text { Multiple symptoms per patient possible. } \\
{ }^{2} \text { Primary disease included colon carcinoma }(n=3) \text {, urinary bladder cancer } \\
(n=3) \text {, pNET }(n=1) \text {, hepatobiliary adenocarcinoma of unknown origin } \\
(n=1) \text {. } \\
{ }^{3} \text { More than one obstructed duodenal part per patient possible. } \\
{ }^{4} \text { More than one oncologic treatment per patient possible. } \\
5 \text { Surgery included a Whipple }(n=2) \text {, pylorus-preserving pancreaticoduode- } \\
\text { nectomy }(n=1) \text { and Roux-en-Y reconstruction }(n=1) \text {. }\end{array}$} \\
\hline
\end{tabular}

ing the opportunity to resume oral intake and three who experienced persistent obstructive complaints were treated with PEG feeding and/or anti-emetics. One of these patients presented at a later stage with a metastatic transverse colon obstruction for which a colon stent was placed. Clinical success was achieved in eight of 10 patients $(80.0 \%)$ with peritoneal carcinomatosis and in 22 of 24 patients (91.7\%) without peritoneal carcinomatosis. Ten of 11 (90.9\%) patients with ascites and 21 of 25 patients (84.0\%) without ascites had clinical success. Clinical success was achieved in six of six patients (100\%) with both peritoneal carcinomatosis and ascites.

\section{Adverse events}

AEs occurred in 12 patients (26.7\%), of which five after stent misplacement. In two patients (4.4\%) AEs were mild, consisting of seeking post-procedural medical consultation for abdominal pain. In one patient this was after accidental puncture of a colonic loop and an aborted procedure; the other returned to the Emergency Room 3 days after a successful EUS-GE. No abnormalities were diagnosed, and both were successfully treated with analgesics and antibiotics. In four patients (8.9\%) AEs were moderate, requiring prolonged hospitalization of 4 to 10 days to administer antibiotics. Antibiotics were administered as prophylaxis after stent misplacement $(n=1)$, due to an E. coli bac- 


\begin{tabular}{|c|c|}
\hline \multicolumn{2}{|l|}{ Year of EUS-GE, n (\%) } \\
\hline . 2015 & $10(22.2)$ \\
\hline . 2016 & $5(11.1)$ \\
\hline . 2017 & $7(15.6)$ \\
\hline - 2018 & $20(44.4)$ \\
\hline - 2019 & $3(6.7)$ \\
\hline \multicolumn{2}{|l|}{ Method of stent placement, $\mathrm{n}(\%)$} \\
\hline - Direct technique & $36(80.0)$ \\
\hline - Balloon-assisted & $9(20.0)$ \\
\hline \multicolumn{2}{|l|}{ Diameter stent, n (\%) } \\
\hline . $15 \mathrm{~mm}$ & $32(72.7)$ \\
\hline . $20 \mathrm{~mm}$ & $12(27.3)$ \\
\hline Balloon dilation, n (\%) & $12(30.8)$ \\
\hline Pre-procedural antibiotics, n (\%) & $18(40.0)$ \\
\hline - $\beta$-lactam & $14(77.8)$ \\
\hline - Other ${ }^{1}$ & $4(22.2)$ \\
\hline Post-procedural antibiotics, n (\%) & $28(62.2)$ \\
\hline - $\beta$-lactam & $22(78.6)$ \\
\hline - Other ${ }^{2}$ & $6(21.4)$ \\
\hline \multicolumn{2}{|c|}{$\begin{array}{l}\text { EUS-GE, endoscopic ultrasound-guided gastroenterostomy } \\
{ }^{1} \text { Other antibiotics prior to EUS-GE: } \beta \text {-lactam + metronidazole }(n=2) \text {, vanco- } \\
\text { mycin }(n=1) \text {, ciprofloxacin }(n=1) \text {. } \\
{ }^{2} \text { Other antibiotics after EUS-GE: } \beta \text {-lactam + metronidazole }(n=4) \text {, vancomy- } \\
\text { cin }(n=1) \text {, ciprofloxacin }(n=1) \text {. }\end{array}$} \\
\hline
\end{tabular}

teraemia $(n=1)$, infection without focus $(n=1)$, and local abdominal pain with elevated inflammatory markers $(n=1)$. The latter three occurred after technically and clinically successful EUS-GE. All patients recovered well. One patient (2.2\%) underwent emergency surgery 13 days after the initial EUS-GE, because of stent dislocation during repeat endoscopy with jejunoscopy through the LAMS. This was considered a severe AE. Five AEs (11.1\%) were fatal, four of which were due to stent misplacement with perforation and abdominal sepsis and one was due to a post-procedural intraperitoneal haemorrhage, noted 24 hours after a technically successful procedure ( Table 4 ).

\section{Survival}

During median overall follow-up of 59 days (IQR 41-128), 32 patients $(71.1 \%)$ died. Causes of death are shown in $>$ Table 3. Median overall survival (OS) was 57 days (IQR 32-114). Median survival of patients after technically successful EUS-GE was 62 days (IQR 41-124), and 34 days (IQR 2-57) after technical failure. Thirteen patients were alive at last contact. Survival is illustrated in > Fig. 2.

\begin{tabular}{|c|c|}
\hline Recurrent obstruction, n (\%) & $2(6.1)$ \\
\hline Technical success, n (\%) & $39(86.7)$ \\
\hline Clinical success, n (\%) & $33(73.3)$ \\
\hline \multicolumn{2}{|l|}{ Diet tolerated after technically successful EUS-GE } \\
\hline - GoOss 0 & $5(12.8)$ \\
\hline - Gooss 1 & $1(2.6)^{1}$ \\
\hline - GOOSS 2 & $15(38.5)$ \\
\hline - GOOSS 3 & $18(46.2)$ \\
\hline Adverse events, $\mathrm{n}(\%)$ & $12(26.7)$ \\
\hline Death, n (\%) & $32(71.1)$ \\
\hline \multicolumn{2}{|l|}{ Cause of death, $n(\%)$} \\
\hline - Progressive disease & $19(59.4)$ \\
\hline - Stent-related & $5(15.6)$ \\
\hline - Other cause & $4(12.5)^{2}$ \\
\hline - Unknown & $4(12.5)$ \\
\hline Median survival after EUS-GE in days (IQR) & $57(32-114)$ \\
\hline $\begin{array}{l}\text { - Median survival after technical successful EUS-GE } \\
\text { (IQR) }\end{array}$ & $62(41-124)$ \\
\hline - Median survival after technical failed EUS-GE (IQR) & $34(2-57)$ \\
\hline Median follow-up in days (IQR) & $59(41-128)$ \\
\hline \multicolumn{2}{|c|}{$\begin{array}{l}\text { EUS-GE, endoscopic ultrasound-guided gastroenterostomy; IQR, interquar- } \\
\text { tile range } \\
{ }^{1} \text { This patient had persistent complaints of occasional vomiting and was not } \\
\text { considered clinically successful. } \\
{ }^{2} \text { Other causes of death include chemotherapy complications, euthanasia, } \\
\text { nosocomial pneumonia, kidney failure. }\end{array}$} \\
\hline
\end{tabular}

\section{Discussion}

This study describes the first European multicenter cohort of patients with malignant GOO who underwent EUS-GE. Recurrent obstruction occurred in two patients (6.1\%) after clinical success, as a result of a narrowed luminal diameter of the LAMS and food impaction. Technical and clinical success was achieved in $86.7 \%$ and $73.3 \%$, respectively. AEs occurred in 12 patients (26.7\%). Median OS was 57 days.

EUS-GE with LAMS is a novel technique to treat malignant GOO. It has the theoretical advantage of a lower risk of dysfunction due to stent migration and tumour ingrowth, because of features such as the biflanged, short length, and fully-covered design, and its positioning outside the tumoral area [16, 21 , 22 ]. In the literature, several studies showed low rates of recurrent obstruction after EUS-GE. These rates varied between $0 \%$ and $4.8 \%$, when defined as recurrent GOO symptoms due to LAMS dysfunction in clinically successful patients [12-14, 16, $19,21,23,24]$. Causes of stent dysfunction included food impaction, inflammatory tissue overgrowth, and stent ingrowth $[12-14,19,23]$. A buried gastric flange of a LAMS has also been reported recently [25]. 
- Table 4 Grading of severity of adverse events after EUS-GE.

\begin{tabular}{|c|c|}
\hline Grading of adverse events & $\begin{array}{l}\mathrm{N} \text { patients } \\
(\%)\end{array}$ \\
\hline \multicolumn{2}{|l|}{ Mild: } \\
\hline - Seeking medical consultation for abdominal pain & $2(4.4)$ \\
\hline \multicolumn{2}{|l|}{ Moderate: } \\
\hline $\begin{array}{l}\text { - Antibiotics administration with 4-10 nights pro- } \\
\text { longed hospitalization }\end{array}$ & $4(8.9)$ \\
\hline \multicolumn{2}{|l|}{ Severe: } \\
\hline - Emergency surgery for stent dislocation & $1(2.2)$ \\
\hline \multicolumn{2}{|l|}{ Fatal: } \\
\hline $\begin{array}{l}\text { - Perforation leading to abdominal sepsis, } n=4 \\
\text { - Post-procedural intraperitoneal haemorrhage, } n=1\end{array}$ & $5(11.1)$ \\
\hline Total & $12(26.7)$ \\
\hline
\end{tabular}

In comparative studies where EUS-GE was compared with duodenal stenting, significantly lower rates of GOO symptom recurrence after EUS-GE were found $[12,13]$. In Ge et al, stent failure requiring repeat intervention occurred in two (8.3\%) LAMS versus $31(32.0 \%)$ duodenal stent placements $(P=$ $0.021)$; calculated per stent placed [13]. In Chen et al, GOO recurrence after clinical success occurred in one (4.0\%) EUS-GE patient versus $10(28.6 \%)$ duodenal stent patients $(P=0.015)$ [12]. When comparing the same 30 EUS-GE patients with 63 SG] patients, long-term patency was not significantly different, with one patient ( $3 \%$ ) experiencing recurrent GOO symptoms after EUS-GE versus nine patients (14\%) after SGJ $(P=0.08)$ [14]. Though the number of patients in these studies was small, data were collected retrospectively and treatment was not allocated randomly, it is suggested that EUS-GE could provide an effective and durable alternative for duodenal stenting and SG] [12-14].

The technical success rate in our study was $86.7 \%$. In previous reports, technical success rates ranged from $86.7 \%$ to $100 \%[12-16,19,23]$. Inclusion of multiple interventional endoscopists could have influenced our rate of technical success, since each endoscopist reported their first EUS-GE cases performed. Five technical failures were due to distal flange misplacement. Four of these cases occurred in a centre in which the first cases were performed with the over-the-guidewire technique, before the free hand technique was applied. The former technique was abandoned later, because it was noted that pushing the guidewire could cause the jejunum to move away from the stomach, resulting in maldeployment of the LAMS [24]. In the fifth case, presence of ascites between the stomach and the jejunum was deemed to preclude technical success.

Our clinical success rate of $73.3 \%$ is lower than previously reported rates of $83.3 \%$ to $95.8 \%$ [12-16, 19, 23]. Inclusion of benign causes of GOO in some of the previous studies may have contributed to higher rates of clinical success, because most of the clinically unsuccessful patients in our study were suspected of having a distal intestinal obstruction or an intestinal motility disorder due to advanced malignancy hindering clinical success $[15,16,19,23]$. In addition, the number of patients with fatal AEs was higher in our study and these patients did not have the chance to regain their ability to eat and become clinically successful.

Our total number of 12 patients with AEs (26.7\%)was higher compared with previously reported rates ranging from $3.5 \%$ to

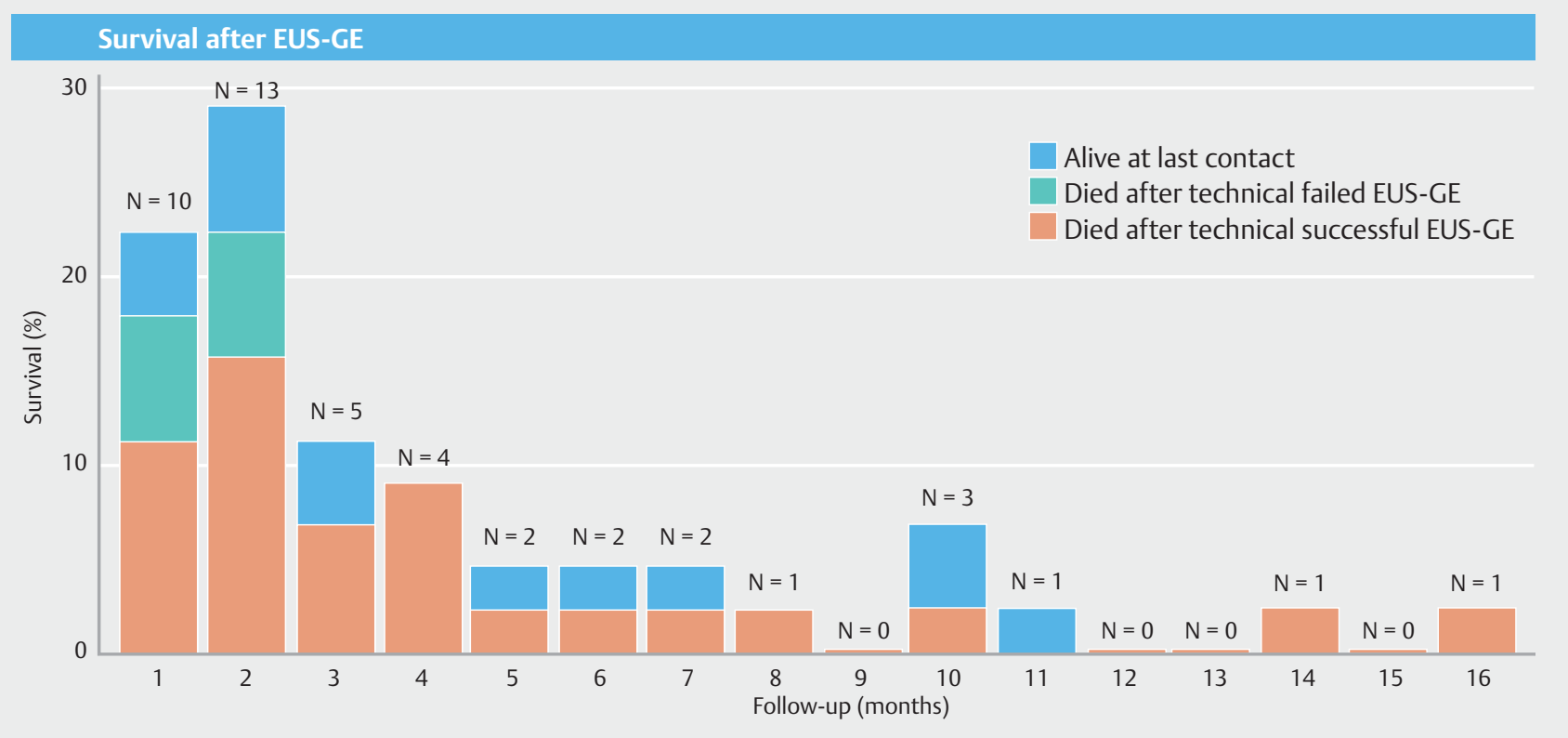

- Fig. 2 Survival after EUS-GE. 
$20.8 \%[12-16,19,23]$. This difference could be explained by the difficulty of retrospective registration of AEs, when available data can be sparse and inconsistent. We found six (13.3\%) mild or moderate AEs that were related to inflammation, antibiotic administration or pain after EUS-GE, which is deemed acceptable after a procedure such as EUS-GE. The stent dislocation during jejunoscopy through the LAMS in one patient, 13 days after the initial EUS-GE, underscores the importance of allowing a mature anastomosis to be formed before it can safely be passed with an endoscope. The number of five fatal AEs in our study, in particular, was higher than in previous reports. Although the percentage of stent misplacements (17.8\%) in our study was in line with the previously reported percentages between $7 \%$ and $36 \%[12-16,19,24]$, it resulted in perforation with fatal peritonitis and abdominal sepsis in 4 of our patients. The fifth patient died from intraperitoneal bleeding after a technically successful procedure. Fatal peritonitis after perforation is a previously reported AE after EUS-GE $[15,16]$. Post-procedural bleeding was also reported previously, but occurred after failed stent placement and was managed successfully with blood transfusions $[15,16]$.

The number of fatal AEs in our study may not reflect contemporary practice but rather individual learning curves and development of technical and clinical practice over recent years. All fatal AEs and most stent misplacements occurred in one center, early after the introduction of EUS-GE. EUS-GE technique has been refined over time (e.g. elimination of guidewire assistance, development of a technique to salvage initial failed placements) and more knowledge about the risks and pitfalls of performing EUS-GE became available [26]. Moreover, interventional endoscopists who began performing EUSGE more recently may have more experience with LAMS, as it is more routinely used for other indications. It highlights, nevertheless, the challenging character and potential risks of EUS-GE, even for experienced interventional endoscopists. Stent misplacement can be fatal, especially in vulnerable and debilitated patients, and pursuing successful stent placements through sufficient training and expertise is crucial.

Our median OS and follow-up were 57 and 59 days, respectively. Recently, Kerdsirichairat et al reported 48 patients with malignant GOO, with a median follow-up of 196 days (IQR 50.5-278.5), the longest follow-up to date [23]. In our study, the duration of follow-up was shorter, partly due to lost contact after referral back to other hospitals or outpatient palliative care, but most importantly due to short survival of our patients. The rate of recurrent obstruction due to LAMS dysfunction was nevertheless low in patients who were followed until death.

We performed an international multicenter study including seven centers from four European countries. All centers used the same type of LAMS (Hot AXIOS stent). We included consecutive cases from the first EUS-GE procedures performed in each centre, to limit selection bias. Treating physicians and general practitioners were contacted to complete follow-up as much as possible and to minimize bias due to missing data.

This study has several limitations. Patients were retrospectively identified, and data were retrospectively collected and interpreted from patient records. Participating centres could have used different selection criteria for EUS-GE, as uniform criteria are currently not available. Patients presenting with malignant GOO who underwent SGJ or duodenal stenting during the study period were not collected and could not be used as comparison group. Our median OS prevents drawing conclusions about long-term patency of LAMS. Due to the palliative care setting, standardized follow-up endoscopies were not performed. Repeat endoscopies were only performed in case of recurrent obstructive symptoms. Therefore, we do not have information on the quality of the LAMS in patients who did not report recurrent obstructive symptoms. Recent reports showed that the coating of the LAMS was degraded in some cases and luminal narrowing of the LAMS has occurred due to hyperplastic tissue ingrowth $[13,27,28]$. LAMS mesh erosion has also been reported [13]. Whether these features could also have occurred in patients who did not report recurrent obstructive symptoms, or were potentially masked by the debilitating course of progressive disease, is unknown. The effect of learning curve on outcomes could not be assessed due to the small number of patients treated by each endoscopist. Although a lower technical success rate was observed in patients with both peritoneal carcinomatosis and ascites, the amount of ascites and peritoneal carcinomatosis and its association with outcomes could not be quantified retrospectively. The size of our cohort did not allow adjustment for patient and procedural characteristics. All but one participating centre were large tertiary centers, possibly hampering generalizability to other centers.

Prospective and comparative studies, ideally with randomized treatment allocation, low rate of attrition, and sufficient duration of follow-up, are warranted to determine the role of EUS-GE in relation to SGJ and duodenal stenting. At this moment, EUS-GE can be considered in patients with a reasonable life expectancy, such as those who typically qualify for SG]. Duodenal stent placement, which carries less risk, remains the preferred treatment in patients with a short life expectancy.

\section{Conclusion}

In conclusion, this international, multicenter, observational cohort study of EUS-GE as treatment of malignant GOO showed low recurrent obstruction rates due to LAMS dysfunction. It suggests that EUS-GE has the potential to be a durable and minimally invasive alternative treatment option, although the limited OS in this cohort does not allow for conclusions to be drawn about long-term outcomes. In addition, EUS-GE remains a challenging technique that requires expertise in LAMS placement and careful implementation in clinical practice, as stent misplacement can lead to serious AEs.

\section{Acknowledgements}

The authors are grateful to Paul Sherliker, Medical Research Council Population Health Research Unit, University of Oxford, for his help constructing the bar chart. 
Dr. Moons, Drs. Masaryk, Dr. Will, Dr. Poley, and Dr. Vleggaar are consultants for Boston Scientific. Dr. Poley is also a consultant for Cook and Pentax. Dr. Perez-Miranda is a consultant for MITech and Boston Scientific, and speaker for Boston Scientific, MITech, Olympus, and Taewoong.

References

[1] Manuel-Vazquez A, Latorre-Fragua R, Ramiro-Perez C et al. Laparoscopic gastrojejunostomy for gastric outlet obstruction in patients with unresectable hepatopancreatobiliary cancers: A personal series and systematic review of the literature. World J Gastroenterol 2018; 24: $1978-1988$

[2] Adler DG, Baron TH. Endoscopic palliation of malignant gastric outlet obstruction using self-expanding metal stents: experience in $36 \mathrm{pa}-$ tients. Am J Gastroenterol 2002; 97: 72-78

[3] Jeurnink SM, Steyerberg EW, van Hooft JE et al. Surgical gastrojejunostomy or endoscopic stent placement for the palliation of malignant gastric outlet obstruction (SUSTENT study): a multicenter randomized trial. Gastrointest Endosc 2010; 71: 490-499

[4] Upchurch E, Ragusa M, Cirocchi R. Stent placement versus surgical palliation for adults with malignant gastric outlet obstruction. Cochrane Database Syst Rev 2018; 5: CD012506

[5] Khashab M, Alawad AS, Shin E] et al. Enteral stenting versus gastrojejunostomy for palliation of malignant gastric outlet obstruction. Surg Endosc 2013; 27: 2068-2075

[6] Maetani I, Tada T, Ukita T et al. Comparison of duodenal stent placement with surgical gastrojejunostomy for palliation in patients with duodenal obstructions caused by pancreaticobiliary malignancies. Endoscopy 2004; 36: 73-78

[7] Uemura S, Iwashita T, Iwata K et al. Endoscopic duodenal stent versus surgical gastrojejunostomy for gastric outlet obstruction in patients with advanced pancreatic cancer. Pancreatology 2018; 18: 601-607

[8] Jang S, Stevens T, Lopez R et al. Superiority of gastrojejunostomy over endoscopic stenting for palliation of malignant gastric outlet obstruction. Clin Gastroenterol Hepatol 2019; 17: 1295-1302

[9] Minata MK, Bernardo WM, Rocha RS et al. Stents and surgical interventions in the palliation of gastric outlet obstruction: a systematic review. Endosc Int Open 2016; 4: E1158-E1170

[10] Mintziras I, Miligkos M, Wachter $S$ et al. Palliative surgical bypass is superior to palliative endoscopic stenting in patients with malignant gastric outlet obstruction: systematic review and meta-analysis. Surg Endosc 2019; 33: 3153-3164

[11] Del Piano M, Ballare M, Montino F et al. Endoscopy or surgery for malignant Gl outlet obstruction? Gastrointest Endosc 2005; 61: 421-426

[12] Chen YI, Itoi T, Baron TH et al. EUS-guided gastroenterostomy is comparable to enteral stenting with fewer re-interventions in malignant gastric outlet obstruction. Surg Endosc 2017; 31: 2946-2952
[13] Ge PS, Young JY, Dong W et al. EUS-guided gastroenterostomy versus enteral stent placement for palliation of malignant gastric outlet obstruction. Surg Endosc 2019; 33: 3404-3411

[14] Khashab MA, Bukhari M, Baron TH et al. International multicenter comparative trial of endoscopic ultrasonography-guided gastroenterostomy versus surgical gastrojejunostomy for the treatment of malignant gastric outlet obstruction. Endosc Int Open 2017; 5: E275E281

[15] Perez-Miranda M, Tyberg A, Poletto D et al. EUS-guided gastrojejunostomy versus laparoscopic gastrojejunostomy: an international collaborative study. J Clin Gastroenterol 2017; 51: 896-899

[16] Tyberg A, Perez-Miranda M, Sanchez-Ocana R et al. Endoscopic ultrasound-guided gastrojejunostomy with a lumen-apposing metal stent: a multicenter, international experience. Endosc Int Open 2016; 4: E276-E281

[17] Garcia-Alonso F], Sanchez-Ocana R, Penas-Herrero I et al. Cumulative risks of stent migration and gastrointestinal bleeding in patients with lumen-apposing metal stents. Endoscopy 2018; 50: 386-395

[18] von Elm E, Altman DG, Egger M et al. The Strengthening the Reporting of Observational Studies in Epidemiology (STROBE) statement: guidelines for reporting observational studies. Ann Intern Med 2007; 147: 573-577

[19] Chen YI, Kunda R, Storm AC et al. EUS-guided gastroenterostomy: a multicenter study comparing the direct and balloon-assisted techniques. Gastrointest Endosc 2018; 87: 1215-1221

[20] Cotton PB, Eisen GM, Aabakken L et al. A lexicon for endoscopic adverse events: report of an ASGE workshop. Gastrointest Endosc 2010; 71: 446-454

[21] Khashab MA, Baron TH, Binmoeller KF et al. EUS-guided gastroenterostomy: a new promising technique in evolution. Gastrointest Endosc 2015; 81: 1234-1236

[22] Khashab MA, Kumbhari V, Grimm IS et al. EUS-guided gastroenterostomy: the first U.S. clinical experience (with video). Gastrointest Endosc 2015; 82: 932-938

[23] Kerdsirichairat T, Irani S, Yang J et al. Durability and long-term outcomes of direct EUS-guided gastroenterostomy using lumen-apposing metal stents for gastric outlet obstruction. Endosc Int Open 2019; 7: E144-E150

[24] Itoi T, Ishii K, Ikeuchi N et al. Prospective evaluation of endoscopic ultrasonography-guided double-balloon-occluded gastrojejunostomy bypass (EPASS) for malignant gastric outlet obstruction. Gut 2016; 65: $193-195$

[25] Dioscoridi L, Forti E, Pugliese F et al. Buried lumen-apposing metal stent after gastrojejunal bypass. Endoscopy 2020; 52: E134-E135

[26] Ligresti D, Amata M, Barresi L et al. The lumen-apposing metal stent (LAMS)-in-LAMS technique as an intraprocedural rescue treatment during endoscopic ultrasound-guided gastroenterostomy. Endoscopy 2019; 51: E331-E332

[27] Madanat L, Saumoy M, Sharaiha RZ. Endoscopic gastrojejunostomy bigger is better. Endoscopy 2018; 50: E331-E332

[28] Parekh P], Shakhatreh MH, Yeaton P. A tale of two LAMS: a report of benign tissue ingrowth resulting in recurrent gastric outlet obstruction. Endosc Int Open 2018; 6: E1390-E1394 